\title{
TANGENTIAL DISPLACEMENT INFLUENCE ON THE CRITICAL NORMAL FORCE OF ADHESIVE CONTACT BREAKAGE IN BIOLOGICAL SYSTEMS
}

UDC (539.612)

\author{
Iakov A. Lyashenko ${ }^{1,2}$ \\ Department of System Dynamics and the Physics of Friction, \\ ${ }^{1}$ Berlin University of Technology, Berlin, Germany \\ ${ }^{2}$ Sumy State University, Sumy, Ukraine
}

\begin{abstract}
The dependencies of the critical components of normal and tangential forces corresponding to the contact breakage between a parabolic indenter and an elastic half-space have been determined taking into account adhesive interaction. In order to describe the adhesive contact, the method of dimensionality reduction (MDR) and the modified rule of Heß taking into account tangential displacements have been used. The influence of the surface energy depending on the indenter separation angle has been studied.
\end{abstract}

Key Words: Adhesion, Friction, Tribology, Shear Force, Numerical Simulation, Method of Dimensionality Reduction

\section{INTRODUCTION}

Adhesive forces play an important role in the processes taking place in biological systems. One bright example of how animals use adhesion is a gecko motion along an inclined surface [1]. During such motion, a firm adhesive contact is established between the gecko's feet and the surface allowing him to move easily both on vertical and horizontal surfaces (on the "ceiling"). This is possible because the gecko's feet surface consists of a huge amount of fibers, each of them having very good adhesive properties. The fibers' elasticity together with their endings' good adhesion to surfaces allows the gecko to easily attach to the majority of natural surfaces. Based on the gecko's feet structure as a natural prototype, a scientific group around S. Gorb [2] has created an artificial material with a similar structure, which "sticks" to almost any surfaces.

Received October 19, 2016 / Accepted November 28, 2016

Corresponding author: Iakov A. Lyashenko

Sumy State University, Department of Modeling of Complex Systems, Rimskii-Korsakova 2, 40007 Sumy, Ukraine

E-mail:nabla04@ukr.net 
It is necessary to emphasize that the adhesive contact can be destroyed not only by an increase in the normal force value, but also by applying a tangential loading as, for example, shown in [3-8]. During the gecko's motion in a horizontal plane (on the "ceiling"), the normal component of the force plays a decisive role. In the case of motion in a vertical plane the normal force required for the foot to detach is created by its muscular power. However, there is also a tangential force caused by gravity. While moving along an inclined plane with different slopes, the contribution of tangential and normal components to the contact breakage will vary. Nevertheless, the gecko's foot detachment always takes place at a particular ratio between these two force components, which is a function of the tangential (or normal) loading [9]. For example, an increasing tangential force will reduce the normal force required to break the contact. Therefore, the aim of the present work is to determine the critical force components that correspond to the adhesive contact breakage taking into account a relationship between the surface energy and the separation angle that was previously determined in [3]. The present research is a continuation of the work [9], in which the surface energy was supposed to be independent of the direction of motion.

It is necessary to point out that we consider a case when there is an equivalent contribution of the tangential and normal loading to the adhesive bonds' breakage. This approach can be applied not only to the gecko's motion description, but also to the adhesion between atomically flat surfaces or long polymer molecules changing their orientation at a tangential displacement. While describing such processes, it is also important to understand what will happen after the adhesive bonds have been broken. For example, in the case of a pure tangential motion, the bonds will be restored after their breakage. However, in the case of a gecko moving along a surface, such behavior is not observed (after separation, the contact is fully recreated but in a different place). That is why we consider the situation when the adhesive bonds are not restored after their destruction. In addition, we limit ourselves to the description of only the contact breakage phase, because we are interested in the dependencies of the critical normal and tangential force components corresponding to the complete contact breakage [9]. Our investigation will be carried out within the framework of the well-known method of dimensionality reduction (MDR) [10], which allows us to reproduce the classical results of the theory by Johnson, Kendall and Roberts [11] (JKR) for the adhesive normal contact using the rule of Hess [10].

The present work consists of two parts. In section 2 we briefly describe the adhesive contact modeling procedure within the MDR. Section 3 will show the investigation of the tangential loading influence and new results are given. Section 4 will be finally dedicated to conclusions.

\section{MDR FOR THE ADHESIVE NORMAL CONTACT}

In order to describe the contact of axially symmetric bodies within the framework of MDR, the following steps should be performed [12]: at first, the initially threedimensional profile $z=f(r)$ is replaced by a one-dimensional function $g(x)$ according to the Abel transform:

$$
g(x)=|x| \int_{0}^{|x|} \frac{f^{\prime}(r)}{\sqrt{x^{2}-r^{2}}} \mathrm{~d} r .
$$


In this paper we restrict ourselves to the parabolic profiles in the form $f(r)=r^{2} /(2 R)$. In this case, Eq. (1) gives the equivalent one-dimensional profile:

$$
g(x)=\frac{x^{2}}{R} .
$$

As a second step, it is necessary to replace the elastic half-space by a one-dimensional elastic foundation of independent linear springs with normal and tangential stiffness:

$$
k_{z}=E^{*} \Delta x, \quad k_{x}=G^{*} \Delta x,
$$

where sampling step $\Delta x$ is the distance between two springs, and effective elastic moduli $E^{*}$ and $G^{*}$ are determined by the equations:

$$
E^{*}=\frac{E}{1-v^{2}}=\frac{2 G}{1-v}, G^{*}=\frac{4 G}{2-v},
$$

With shear modulus $G$ and Poisson number $v$, leading to the so-called Mindlin ratio:

$$
\frac{G^{*}}{E^{*}}=\frac{2-2 v}{2-v} .
$$

Later we will use a criterion of detachment given by Eq. (14), which contains equivalent contributions to the adhesive force from both the normal and tangential displacements of the indenter. We should note that Eq. (14) is valid only for $v=0$. In this case, the stress concentration factors for the modes $I I$ and $I I I$ are equal along the entire boundary line [9, 13]. On the other hand, for a no-slip contact between a rigid indenter and an elastic half space, the condition of elastic similarity (which necessarily has to be fulfilled to ensure the exact correctness of the MDR) corresponds to $v=0.5$. However, accounting for elastic dissimilarity will severely complicate the calculations and the error made by not accounting for it was proven to be small [14]. Therefore, in further calculations we have chosen $v=0$ in order to provide the correctness of the detachment criterion in Eq. (14). The Mindlin ratio in this case will be equal to one.

If transformed profile $g(x)$ is pressed into the elastic foundation with penetration depth $d$, the displacement of an individual spring inside the elastic contact will be determined by the following expression:

$$
u_{z}(x)=d-g(x)=d-\frac{x^{2}}{R} .
$$

The adhesive contact size (i.e. its radius $a$ ) can be easily found using the rule of Heß, which gives the tension level for the boundary springs in contact $\Delta l=-u_{z}(a)$, where the value of $\Delta l$ is determined by the following equation [12]:

$$
\Delta l=\sqrt{\frac{2 \pi a \Delta \gamma}{E^{*}}} .
$$


By combining expressions (6) and (7), we get the equation:

$$
d=\frac{a^{2}}{R}-\sqrt{\frac{2 \pi a \Delta \gamma}{E^{*}}} .
$$

As a result, total normal force value $F_{z}$ can be calculated as the sum of forces of all individually stretched and compressed springs [10]:

$$
F_{z}(a)=\int_{-a}^{a} u_{z}(x) \mathrm{d} x=2 E^{*} \int_{0}^{a}\left(d-\frac{x^{2}}{R}\right) \mathrm{d} x=\frac{4 E^{*} a^{3}}{3 R}-\sqrt{8 \pi a^{3} E^{*} \Delta \gamma} .
$$

Let us now consider a more general case when the indenter also moves in the tangential direction with displacement $u_{x}^{(0)}$. For convenience, we represent our results in terms of the following dimensionless parameters:

$$
\tilde{a}=\frac{a}{a_{0}}, \quad \tilde{F}_{z}=\frac{F_{z}}{F_{0}}, \quad \tilde{d}=\frac{d}{d_{0}}, \quad \tilde{u}_{x}^{(0)}=\frac{u_{x}^{(0)}}{d_{0}}, \tilde{u}_{z}=\frac{u_{z}}{d_{0}},
$$

where $F_{0}, a_{0}$ and $d_{0}$ are the critical values of the normal force, the contact radius and the absolute value of the indentation depth at the moment of the parabolic indenter's detachment from the elastic half-space under "fixed load" conditions [15]:

$$
F_{0}=\frac{3}{2} \pi R \Delta \gamma, \quad a_{0}=\left(\frac{9 \pi R^{2} \Delta \gamma}{8 E^{*}}\right)^{1 / 3}, \quad d_{0}=\left(\frac{3 \pi^{2} R \Delta \gamma^{2}}{64 E^{* 2}}\right)^{1 / 3} .
$$

In terms of these dimensionless parameters Eqs. (8) and (9) take the form:

$$
\begin{gathered}
\tilde{d}=3 \tilde{a}^{2}-4 \tilde{a}^{1 / 2}, \\
\tilde{F}=\tilde{a}^{3}-2 \tilde{a}^{3 / 2},
\end{gathered}
$$

which, of course, just reproduce the JKR solution [11].

\section{INFLUENCE OF TANGENTIAL DISPLACEMENT}

Let us consider a situation of an actually non-zero tangential displacement $u_{x}^{(0)}$. In this case, the energy released during the detachment of the two outermost springs will be equal to $E^{*} u_{z}(a)^{2} \Delta x+G^{*} u_{x}^{(0) 2} \Delta x$. By equalizing it with the adhesive work $2 \pi a \Delta x \Delta \gamma$, we get the equilibrium condition in the form [9]:

$$
E^{*} u_{z}(a)^{2}+G^{*} u_{x}^{(0) 2}=2 \pi a \Delta \gamma .
$$

In our previous work, we supposed that adhesive work $\Delta \gamma$ is independent of the tangential loading [9]. However, some investigations have shown that such dependence may occur [3, 4, 16]. For example, in [3] in order to take into account the influence of the tangential displacement, the surface energy dependence has been proposed in the form: 


$$
\gamma_{0}=\Delta \gamma\left(1+\frac{E^{*}}{G^{*}} \tan ^{2}\left[(1-\lambda) \tan ^{-1}\left(\frac{G^{*} u_{x}^{(0)}}{E^{*} u_{z}(a)}\right)\right]\right),
$$

with dimensionless parameter $\lambda$, introduced in order to determine how the surface energy depends on the motion direction. This equation has been obtained in the work [3] for the crack opening regime; that is why it is valid only for negative normal forces values $\tilde{F}_{z}$ (or in our notations for negative displacement values $\left.\tilde{u}_{z}(\tilde{a})\right)$. The situation of a surface energy independent of the motion direction is determined by $\lambda=1$. In order to find out how $\lambda$ influences the contact breakage process, we should rather use energy $\gamma_{0}$ (Eq. (15)) in Eq. (14) instead of standard constant $\Delta \gamma$.

Using dimensionless parameters, the corresponding equilibrium condition takes the form:

$$
\frac{E^{*}}{G^{*}} \tilde{u}_{z}(\tilde{a})^{2}+\tilde{u}_{x}^{(0)^{2}}=16 \tilde{a} \frac{E^{*}}{G^{*}}\left(1+\frac{E^{*}}{G^{*}} \tan ^{2}\left[(1-\lambda) \tan ^{-1}\left(\frac{G^{*} \tilde{u}_{x}^{(0)}}{E^{*} \tilde{u}_{z}(\tilde{a})}\right)\right]\right) \text {. }
$$

Let us perform numerical simulations of the adhesive contact using this detachment condition at different values for $\lambda$. In the case under consideration, function $\tilde{u}_{z}(\tilde{a})$ is determined by expression (16). Using Eq. (6), we can find the relationship between indentation depth $d$ and contact radius $a$ in the form:

$$
d=\frac{a^{2}}{R}+u_{z}(a)
$$

The normal and tangential forces are functions of contact radius $a$ :

$$
\begin{gathered}
F_{z}=2 E^{*}\left(a d-\frac{a^{3}}{3 R}\right), \\
F_{x}=2 G^{*} a \cdot u_{x}^{(0)} .
\end{gathered}
$$

In terms of the dimensionless parameters in Eq. (10), Eqs. (17)-(19) can be written in the following form:

$$
\begin{aligned}
& \tilde{d}=3 \tilde{a}^{2}+\tilde{u}_{z}(\tilde{a}), \\
& \tilde{F}_{z}=\frac{\tilde{a}}{2}\left(\tilde{d}-\tilde{a}^{2}\right), \\
& \tilde{F}_{x}=\frac{G^{*}}{2 E^{*}} \tilde{a} \cdot \tilde{u}_{x}^{(0)} .
\end{aligned}
$$

These equations define the dependencies of the normal force on the indentation depth taking into account the tangential displacement. It is necessary to point out that substitution of the indentation depth (Eq. (20)) into the equation for the normal force (Eq. (21)) at zero tangential displacement $\tilde{u}_{x}^{(0)}=0$ leads to the classical solution (Eq. (13)) for the normal contact. 
Let us consider "fixed-grips" and "fixed-loads" loading conditions. In the case of "fixed-grips" conditions, a very stiff external system controls the macroscopic indenter displacement. Physically it means that during the system's motion toward the equilibrium state, the displacement value is kept constant. The "fixed load" conditions can be implemented physically with the help of a very soft spring. As a result, the force value is fixed during the relaxation process. We note that the MDR-relations described before have been successfully applied to the simulation of the adhesion influence at particles elastic collisions under "fixed-grips" loading conditions [17].

In the case of "fixed-grips", the loss of the contact stability is defined by expression $\mathrm{d} \tilde{d}(a) / \mathrm{d} \tilde{a}=0$, which using Eq. (20) can be written in the explicit form:

$$
\frac{\mathrm{d} \tilde{u}_{z}}{\mathrm{~d} \tilde{a}}+6 \tilde{a}=0 .
$$

By solving the system of Eqs. (16) and (23), we have obtained the dependence of critical radius $\tilde{a}_{c, f g}$ on tangential displacement $\tilde{u}_{x}^{(0)}$. The substitution of the result into Eqs. (20)-(22) allows us to obtain the desired relationship between the normal force (the adhesion force) and applied tangential force $\tilde{F}_{z}\left(\tilde{F}_{x}\right)$.

Fig. 1a shows these dependencies built for different values of $\lambda$. Note that at zero tangential force $\tilde{F}_{x}=0$ (or zero displacement $\tilde{u}_{x}^{(0)}=0$ ) under "fixed-grips" conditions, the critical normal force is $\tilde{F}_{z}(0)=-5 / 9$ for all of the curves [9].

Under the "fixed-load" conditions, the instability occurs when the negative normal force reaches its maximum value [10]. Consequently, the instability condition is written in the form $\mathrm{d} \tilde{F}_{z} / \mathrm{d} \tilde{a}=0$, resulting in the equation:

$$
\frac{\tilde{u}_{z}}{\tilde{a}}+\frac{\mathrm{d} \tilde{u}_{z}}{\mathrm{~d} \tilde{a}}+6 \tilde{a}=0 .
$$

We have performed the above described numerical analysis. But instead of Eq. (23) we have used expression (24). Fig. 1b gives the numerical calculations results. Note that all the curves in Fig. 1 are shown in the range of negative forces $\tilde{F}_{z}<0$, for which Eq. (15) holds true. In Fig. 1b the critical value of the normal force without the tangential displacement is $\tilde{F}_{z}(0)=-1$.

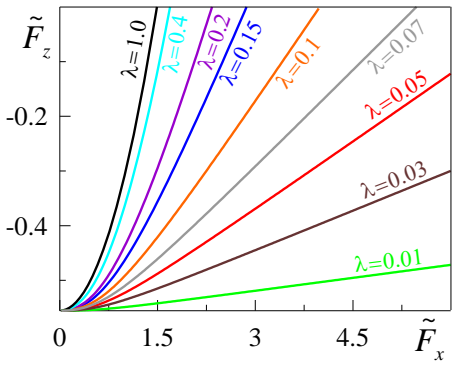

a)

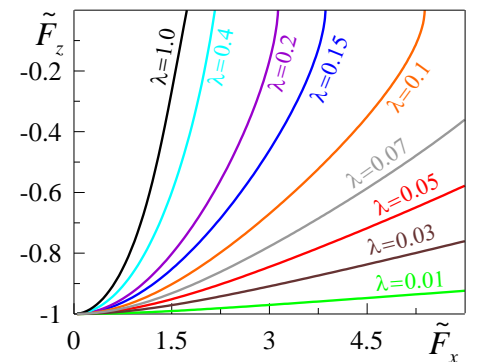

b)

Fig. 1 Normalized dependencies of critical normal force $\tilde{F}_{z}$ on tangential force $\tilde{F}_{x}$ for $E^{*}=G^{*}$ at different values of $\lambda$ : (a) "fixed-grips" loading conditions in both directions; (b) "fixed-load" conditions in the vertical direction and "fixed-grips" conditions in the tangential direction 
In accordance with the results shown in Fig. 1 in both the considered cases the indenter can detach from the half space at zero normal force at the expense of tangential force $\tilde{F}_{x}$. These critical tangential forces versus parameter $\lambda$ at zero normal forces $\tilde{F}_{z}=0$ are shown in Fig. 2 for "fixed-grips" conditions (solid curve) and "fixed-load" conditions (dashed curve). Both the curves correspond to the results shown in Fig. 1. It can be seen from Fig. 2 that these two dependencies $\tilde{F}_{x}(\lambda)$ have the similar form, but under the "fixed-grips" conditions, the critical value of tangential force required for detaching is smaller than for situations with "fixed load" conditions.

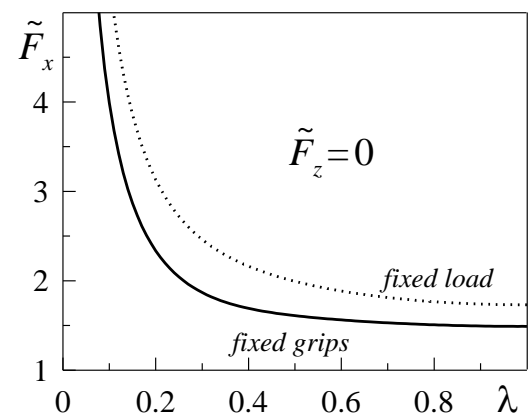

Fig. 2 Normalized dependencies of critical tangential force $\tilde{F}_{x}$ on $\lambda$ at zero value of normal force for the parameters of Fig. 1a (solid curve) and Fig. 1b (dashed curve).

\section{CONCLUSIONS}

The adhesive contact between an axially-symmetric indenter and an elastic half-space has been investigated under superimposed normal and tangential loading taking into account the dependence of the surface energy on the separation direction. It has been shown that the presence of the tangential displacement leads to a decrease in the critical value of the normal force that corresponds to detachment of the indenter from the surface. Different combinations of "fixed-load" and "fixed-grips" loading conditions have been studied in the normal and tangential direction. For each case, the universal curves have been built in terms of corresponding dimensionless parameters. The investigation results can be applied to the description and simulation of adhesive processes taking place at the adhesive interaction of a gecko's foot with a surface along which it moves.

Acknowledgements: The presented work has been performed during scientific visit of the author to Institute of Mechanics (Berlin University of Technology). The author is grateful to Prof. V. L. Popov for the invitation, for the financial support of the work, for the formulation of the problem and his helpful advices during the investigation process. This work was partially supported by Ministry of Education and Science of Ukraine under the project No. 0116 U006818 "Thermodynamic theory of the phase transitions between structural states of the boundary lubricant with spatial inhomogeneity". 


\section{REFERENCES}

1. Gao, H., Wang, X., Yao, H., Gorb, S., Arzt, E., 2005, Mechanics of hierarchical adhesion structures of geckos, Mechanics of Materials, 37(2-3), pp. 275-285.

2. Carbone, G., Pierro, E., Gorb, S. N., 2011, Origin of the superior adhesive performance of mushroomshaped microstructured surfaces, Soft Matter, 7(12), pp. 5545-5552.

3. Hutchinson, J. W., Suo, Z., 1991, Mixed mode cracking in layered materials, Advances in Applied Mechanics, 29, pp. 63-191.

4. Waters, J. F., Guduru, P. R., 2010, Mode-mixity-dependent adhesive contact of a sphere on a plane surface, Proc. Royal Soc. A, 466(2117), pp. 1303-1325.

5. Waters, J. F., Guduru, P. R., 2011, A mechanism for enhanced static sliding resistance owing to surface waviness, Proc. Royal Soc. A, 467(2132), pp. 2209-2223.

6. Waters, J. F., Kalow, J., Gao, H., Guduru, P. R., 2012, Axisymmetric adhesive contact under equibiaxial stretching, The Journal of Adhesion, 88(2), pp. 134-144.

7. Waters, J. F., Gao, H. J., Guduru, P. R., 2011, On adhesion enhancement due to concave surface geometries, The Journal of Adhesion, 87(3), pp. 194-213.

8. Popov, V. L., Dimaki, A. V., 2016, Friction in an adhesive tangential contact in the Coulomb-Dugdale approximation, The Journal of Adhesion, doi: 10.1080/00218464.2016.1214912

9. Popov, V. L., Lyashenko, I. A., Filippov, A. E., 2016, Influence of tangential displacement on the force of adhesion between a parabolic profile and plane surface, arXiv: 1611.00570 [cond-mat.soft], $12 \mathrm{pp}$

10. Popov, V. L, Heß, M., 2015, Method of dimensionality reduction in contact mechanics and friction, Springer, $265 \mathrm{p}$.

11. Johnson, K. L., Kendall, K., Roberts, A. D., 1971, Surface energy and the contact of elastic solids, Proc. Royal Soc. Lond. A, Mathematical and Physical Sciences, 324(1558), pp. 301-313.

12. Popov, V. L., 2013, Method of reduction of dimensionality in contact and friction mechanics: A linkage between micro and macro scales, Friction, 1(1), pp. 41-62.

13. Johnson, K. L., 1997, Adhesion and friction between a smooth elastic spherical asperity and a plane surface, Proc. R. Soc. Lond. A, 453, pp. 163-179.

14. Borodich, F.M., Galanov, B.A., Prostov, Y.I., Suarez-Alvarez, M.M., 2012, Influence of Complete Sticking on the Indentation of a Rigid Cone into an Elastic Half Space in the Presence of Molecular Adhesion. Journal of Applied Mathematics and Mechanics, 76, pp. 590-596.

15. Popov, V. L., 2010, Contact mechanics and friction. Physical principles and applications, Springer, 361p.

16. Kim, K. -S., McMeeking, R. M., Johnson, K. L., 1998, Adhesion, slip, cohesive zones and energy fluxes for elastic spheres in contact, Journal of the Mechanics and Physics of Solids, 46(2), pp. 243-266.

17. Lyashenko, I. A., Willert, E., Popov, V. L., 2016, Adhesive impact of an elastic sphere with an elastic half space: Numerical analysis based on the method of dimensionality reduction, Mechanics of Materials, 92, pp. 155-163. 